\title{
Design and Simulation Verification of New Miniaturized Dual-frequency Microstrip Antenna
}

\author{
An-Hong Tian, Cheng-Biao Fu, and Shun-Ji Zhang ${ }^{* *}$ \\ College of Information Engineering, Qujing Normal University, Qujing, Yunnan 655011, China
}

(Received March 28, 2018; accepted August 24, 2018)

Keywords: dual-frequency microstrip antenna, resonant frequency, return loss, radiation direction

Because of the problem that the global positioning system (GPS) fails to locate subjects owing to the occlusion of satellite signals in some environments, a combined navigation and positioning scheme based on GPS and digital television multimedia broadcasting (DTMB) was proposed. The design process of a dual-frequency microstrip antenna was described in detail. Our simulation showed that the return loss was less than $-10 \mathrm{~dB}$, and the antenna had three resonance points, namely, $0.69,1.55$, and $1.78 \mathrm{GHz}$, with working frequency bands of $0.68-0.70,1.54-1.58$, and $1.77-1.79 \mathrm{GHz}$, respectively. These results proved that the dualfrequency microstrip antenna could simultaneously receive GPS and DTMB signals, and that the antenna was a circularly polarized antenna with single-point feeding and excellent radiation characteristics and strong practicability. In addition, the particle filter (PF) algorithm was applied to the combined positioning scheme, and the dynamic tracking effect of such an algorithm was simulated under different particle numbers. Simulation results showed that the filter tracking trajectory was close to the real trajectory, and that the higher the number of particles, the higher the positioning accuracy. This research verified the feasibility of navigation and positioning based on GPS and DTMB.

\section{Introduction}

The global positioning system (GPS) fails to locate subjects owing to the occlusion of signals in some areas, such as cities, tunnels, canyons, indoors, and underground parking areas. Currently, wireless positioning systems tend to fuse multiple signal sources to form a combined positioning system. Because of China's independently developed digital television multimedia broadcasting (DTMB) standard, which has advantages of high positioning accuracy, high signal strength, strong penetrability, and wide coverage,$^{(1,2)}$ in this paper, a combined navigation positioning system based on GPS and DTMB is proposed to solve the blind zone problem of a GPS single satellite system. However, GPS and DTMB signals work in different frequency bands. The premise of combined positioning is that the receiver can simultaneously receive GPS and DTMB signals, but there are few reports on antennas of different combined positioning systems. Therefore, it is of great significance to study a dual-frequency antenna that

\footnotetext{
*Corresponding author: e-mail: fucb@mail.qjnu.edu.cn

** Corresponding author: e-mail: ykmz56@126.com

https://doi.org/10.18494/SAM.2018.2068
} 
can simultaneously receive GPS and DTMB signals. This premise is the basis for implementing combined navigation and tracking.

At present, there are many types of antenna used by receivers, including monopole antennas, dipole antennas, and microstrip antennas. ${ }^{(3,4)}$ The performance characteristics of these antennas are different, and it is necessary to consider the performance of receivers to select antennas practically. Microstrip antennas have become the most suitable antennas of receivers owing to their small size and excellent performance. There are mainly five types of multifrequency technology for microstrip antennas, namely, etching slits near the radiating edge of a rectangular patch, using a multilayer patch, a slot-coupled rectangular patch, a rectangular patch with a shorting pin and a slit, and dual-frequency technology with a single feed and a single layer. ${ }^{(5,6)}$ Sheta et al. proposed an H-shaped microstrip antenna, which had the advantages of miniaturization and multiband operation compared with a conventional rectangular microstrip patch antenna. ${ }^{(7)}$ Nasiri et al. designed a frequency-reconfigurable microstrip patch antenna, which realized a frequency tuning range of $2.3 \mathrm{GHz}{ }^{(8)}$ Caratelli et al. loaded Z-grooves on the basis of E-grooves, realizing a broadband slot microstrip antenna with a bandwidth of $29.8 \%$, but the processing technique used was complicated. ${ }^{(9)}$ In our current study, a dual-frequency antenna that can receive GPS and DTMB signals simultaneously is designed. It has a simple structure and is easy to manufacture and debug. It simultaneously receives multiple positioning signal sources, ensures the feasibility of navigation combined multisource signals, and helps to increase the number of observation satellites. This study improves positioning accuracy, avoids the constraints of a single positioning system, and proves the feasibility of combined positioning based on GPS and DTMB.

\section{Materials and Methods}

\subsection{Combined positioning system}

A pseudo-range method has been adopted in the combined positioning system based on GPS and DTMB. There are two different positioning system models in this system, with each positioning system model having its own clock. Thus, there are at least five unknown parameters, which include three-dimensional position coordinates and two system clock deviations. The combined positioning equation is expressed as

$$
\left\{\begin{array}{c}
\rho_{1}=\sqrt{\left(x_{1}-x_{u}\right)^{2}+\left(y_{1}-y_{u}\right)^{2}+\left(z_{1}-z_{u}\right)^{2}}+k_{1} b_{1}+k_{2} b_{2}, \\
\rho_{2}=\sqrt{\left(x_{2}-x_{u}\right)^{2}+\left(y_{2}-y_{u}\right)^{2}+\left(z_{2}-z_{u}\right)^{2}}+k_{1} b_{1}+k_{2} b_{2}, \\
\rho_{3}=\sqrt{\left(x_{3}-x_{u}\right)^{2}+\left(y_{3}-y_{u}\right)^{2}+\left(z_{3}-z_{u}\right)^{2}}+k_{1} b_{1}+k_{2} b_{2}, \\
\quad \cdots \\
\rho_{n}=\sqrt{\left(x_{n}-x_{u}\right)^{2}+\left(y_{n}-y_{u}\right)^{2}+\left(z_{n}-z_{u}\right)^{2}}+k_{1} b_{1}+k_{2} b_{2},
\end{array}\right.
$$


where $\left(x_{i}, y_{i}, z_{i}\right)$ indicates the coordinate information of the GPS satellite or DTMB television tower, $\rho_{i}$ has a pseudo-range value, $\left(x_{u}, y_{u}, z_{u}\right)$ indicates unknown three-dimensional coordinate information, $b_{1}$ is the clock offset of GPS, $b_{2}$ is the clock offset of DTMB, and $b_{1}=c \cdot \Delta t_{G P S}$, $b_{2}=c \cdot \Delta t_{D T M B}$. In the presence of a GPS signal, $k_{1}=1$, otherwise, $k_{2}=1$.

\subsection{Dual-frequency microstrip antenna design index}

It is well known that two transmission frequencies of 1575.42 and $1227.60 \mathrm{MHz}$ are used in GPS navigation satellites. However, according to China's current radio-frequency allocation plan, the working frequency band of a digital television terrestrial broadcasting system is $470-860 \mathrm{MHz}$, and according to the opinions of relevant foreign experts, terrestrial digital television broadcasting with mobile reception as its main business is being carried out, with a working frequency generally between 550 and $700 \mathrm{MHz}$. Thus, the dual-frequency antenna selects the $1.57 \mathrm{GHz}$ frequency of GPS and the $0.69 \mathrm{GHz}$ frequency of DTMB. The return loss is less than $-10 \mathrm{~dB}$.

\subsection{Selection of dielectric substrate material and calculation of radiant panel size}

Since the microstrip antenna has inherent disadvantages of relatively small bandwidth, in order to obtain the characteristics of wide frequency, we should select a dielectric constant with a relatively small but thick medium. In the simulation of this study, the selected medium was Rogers RT/duroid 5880 (tm), which has a basic thickness of $3 \mathrm{~mm}$ and a relative dielectric constant of 2.2 .

The size of the radiant panel is calculated as

$$
\begin{gathered}
W=\frac{c}{2 f_{r}}\left(\frac{\varepsilon_{r}+1}{2}\right)^{-\frac{1}{2}}, \\
L=\frac{c}{2 f_{r} \sqrt{\varepsilon_{e}}}-2 \Delta L, \\
\varepsilon_{e}=\frac{\varepsilon_{r}+1}{2}+\frac{\varepsilon_{r}-1}{2}\left(1+\frac{10 h}{W}\right)^{-1 / 2}, \\
\Delta L=0.412 h \frac{\left(\varepsilon_{e}+0.3\right)(W / h+0.264)}{\left(\varepsilon_{e}-0.258\right)(W / h+0.8)},
\end{gathered}
$$

where $f_{r}$ is the resonant frequency, $W$ is the width of the radiating element, $L$ is the length of the radiating element, $h$ is the thickness of the dielectric substrate, $\Delta L$ is the degree of line elongation, $c$ is the speed of light in vacuum, $\varepsilon_{e}$ is the equivalent dielectric constant, and $\varepsilon_{r}$ is the relative dielectric constant. 


\subsection{Optimization of microstrip antenna size}

It can be seen from Eqs. (2) and (3) that the higher the resonance frequency, the smaller the size of the antenna. To reduce the size of the microstrip antenna, the microstrip antenna size has been calculated by considering the resonant frequency of $1.575 \mathrm{GHz}$, and then short-circuit pin loading has been adopted to realize the low resonant frequency of the antenna, so that the microstrip antenna has the characteristic of dual frequency, allowing it to simultaneously receive GPS and DTMB signals.

Combined with the above various factors, a circularly polarized antenna with single-point feeding has been designed, and the final optimized antenna is obtained in accordance with the above formula. The radiation plate size, grounding plate size, and thickness of the microstrip antenna are $75.2 \times 75.2 \mathrm{~mm}^{2}, 95.2 \times 95.2 \mathrm{~mm}^{2}$, and $3 \mathrm{~mm}$, respectively.

\subsection{Structural design of microstrip antenna}

In line with the above design parameters, the antenna used in this study is designed for the resonant frequency of $1.575 \mathrm{GHz}$, and then the short-circuit pin is used to achieve the dualfrequency operation. The designed microstrip antenna structure is shown in Fig. 1.

\section{Experimental Results and Discussion}

\subsection{Simulation model of microstrip antenna}

Ansoft HFSS software was selected to simulate the designed antenna in this paper. Firstly, the microstrip antenna simulation structure diagram was drawn on the basis of its design structure diagram. Secondly, we set the materials of the microstrip antenna; the radiant panel and grounding plate were determined to be good conductors, shorting pins were copper metal,

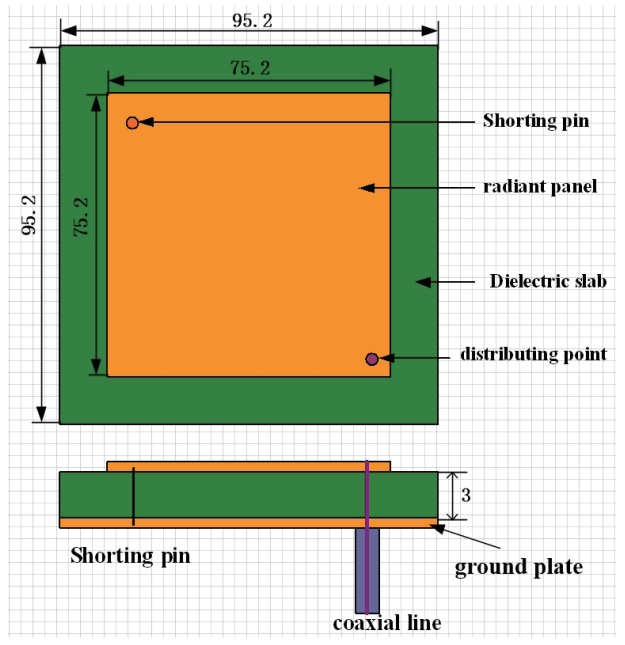

Fig. 1. (Color online) Structure of microstrip antenna (unit: $\mathrm{mm}$ ). 
and the relative dielectric constant was 2.2. Afterwards, the radiation boundary size of the microstrip antenna was defined as the space of $700 \times 700 \times 270 \mathrm{~mm}^{3}$. Finally, the simulation parameters of the microstrip antenna were selected; that is, the wave port feed mode was selected, and the simulation sweep frequency range was $0.30-2.00 \mathrm{GHz}$. The simulation model of the microstrip antenna is shown in Fig. 2.

\subsection{Simulation results for microstrip antenna}

The return loss diagram and radiation pattern of the antenna are shown in Figs. 3-5, respectively. As shown in Fig. 3, when the return loss was less than $-10 \mathrm{~dB}$, the antenna had three resonance points, namely, $0.69,1.55$, and $1.78 \mathrm{GHz}$, with working frequency bands of $0.68-0.70,1.54-1.58$, and $1.77-1.79 \mathrm{GHz}$, respectively. Therefore, the antenna could simultaneously receive GPS and DTMB signals.

From Fig. 4, when the resonant frequency of the antenna was $0.69 \mathrm{GHz}$, the gains of the E-plane $\left(0^{\circ}\right)$ and $\mathrm{H}$-plane $\left(90^{\circ}\right)$ for the antenna were 13.25 and $16.25 \mathrm{~dB}$, and the corresponding main radiation directions were $0^{\circ}$ and $\pm 30^{\circ}$, respectively. The $3 \mathrm{~dB}$ main lobe radiation angle of the E-plane for the antenna was between $\pm 30^{\circ}$, and the $3 \mathrm{~dB}$ main lobe radiation angle of the H-plane was between $\pm 45^{\circ}$. Therefore, when the antenna was operated at the low-frequency end, its E-plane and H-plane radiation beam angles were higher, and such an antenna could be used as a receiving antenna.

It could be seen from Fig. 5 that when the operating frequency of the antenna was $1.575 \mathrm{GHz}$, the E-plane and H-plane gains of the antenna were both $16.890 \mathrm{~dB}$, and the main radiation directions were both $0^{\circ}$. The $3 \mathrm{~dB}$ main lobe radiation angle of the E-plane for the antenna was in the ranges of $-10^{\circ}-5^{\circ},-85^{\circ}--75^{\circ}$, and $75-90^{\circ}$, and the $3 \mathrm{~dB}$ main lobe radiation angle of the H-plane was in the ranges of $-8^{\circ}-5^{\circ},-30^{\circ}--25^{\circ}$, and $-95^{\circ}--80^{\circ}$. Thus, when the antenna was operated at the high-frequency end, the E-plane and H-plane radiation beam angles of the antenna were relatively low. The antenna had several radiation beams, a good directional radiation beam, and a higher gain; thus, it could be used as a transmitting antenna.

Furthermore, the gain of the microstrip antenna at each azimuth solid angle for two resonant operating frequencies was greater than $3 \mathrm{~dB}$, except for spatial solid angles between 120 and

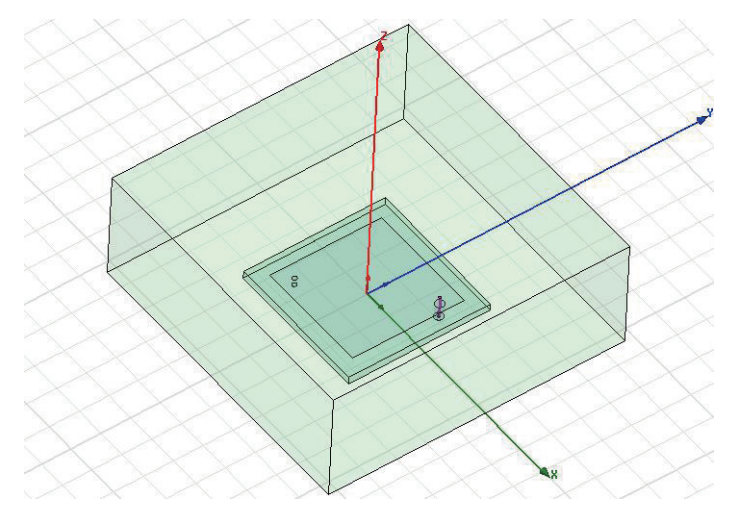

Fig. 2. (Color online) Simulation model of microstrip antenna. 


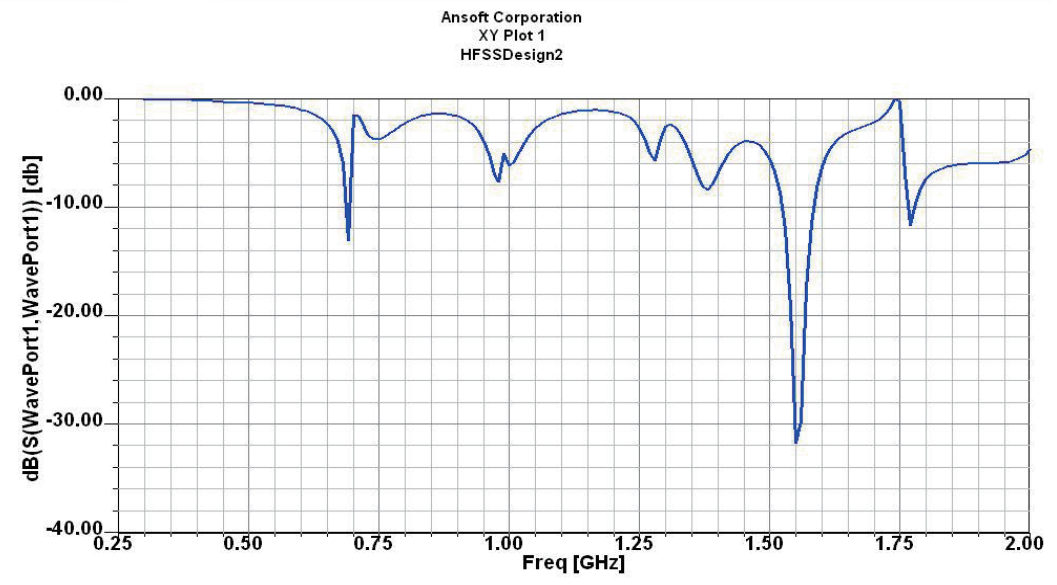

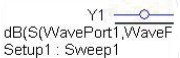

Fig. 3. (Color online) Simulated antenna return loss.

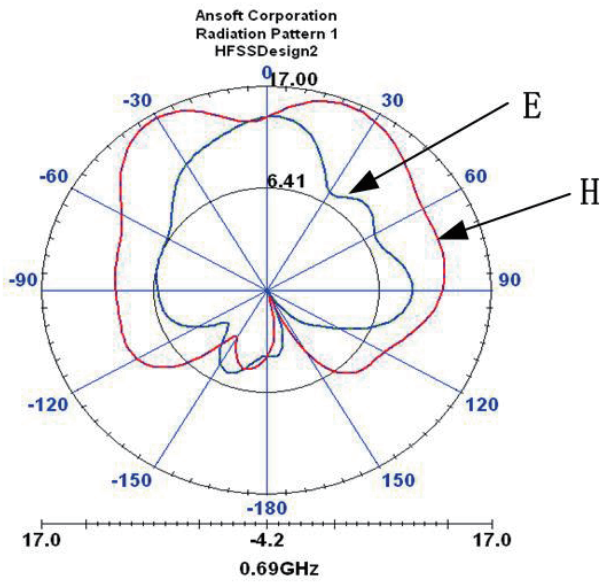

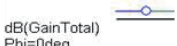
Phi=odeg

dBB(GainTotal) Phi=90deg
Setup 1: Sweep 1

Fig. 4. (Color online) Simulated antenna radiation pattern for $0.69 \mathrm{GHz}$.
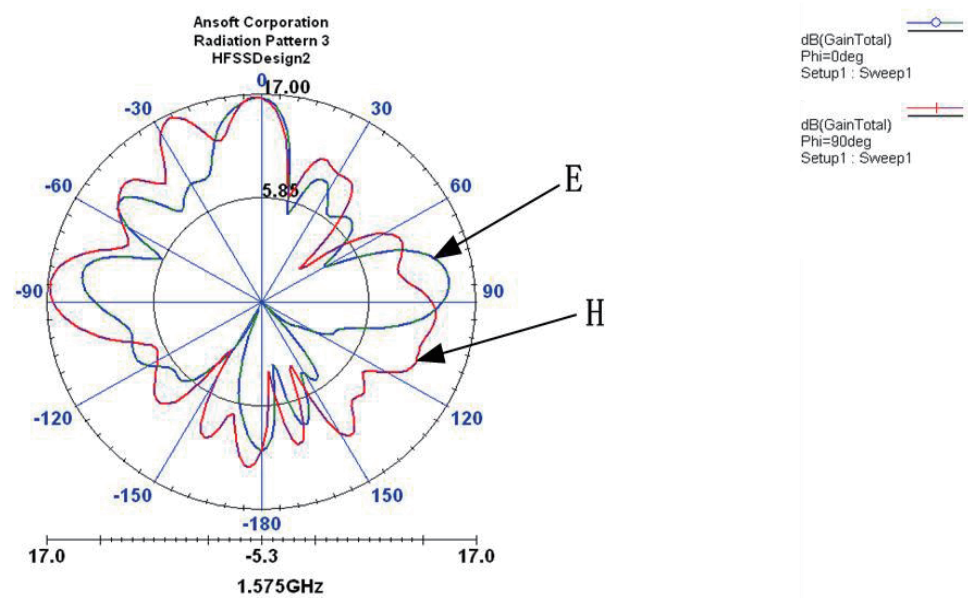

Fig. 5. (Color online) Simulated antenna radiation pattern for $1.575 \mathrm{GHz}$. 
$80^{\circ}$ at the low-frequency end, which could effectively receive the spatial incoming wave signal, revealing that the antenna had good reception performance.

It can be clearly seen from the above simulation that the dual-frequency antenna designed in this study can receive signals for the two frequencies of 0.69 and $1.575 \mathrm{GHz}$, and that the antenna also has good radiation characteristics and high practicability. These results validate the premise of the GPS- and DTMB-based combined positioning model used in this study, and confirms that there are antennas that can simultaneously receive GPS and DTMB signals.

\subsection{Location tracking results of particle filter (PF) algorithm}

The moving target model studied in this paper was a plane variable turning model in the three-dimensional state. The velocity components of the target object in the $X-, Y$-, and $Z$-directions were 7,7 , and $7 \mathrm{~m} / \mathrm{s}$, and the acceleration components were $0.5,-0.5$, and $-0.5 \mathrm{~m} / \mathrm{s}^{2}$, respectively. The noise obeyed a Gaussian distribution with a mean of zero, the noise mean square error was $0.01 \mathrm{~m} / \mathrm{s}^{2}$, and the standard deviation of the distance error was $7 \mathrm{~m}$. The simulation time was set to $100 \mathrm{~s}$ and the time step was $1 \mathrm{~s}$. The initial coordinates of the target object were determined to be $(2,2,2)$. The initial state error covariance matrix was zero. The dynamic tracking effect of the PF algorithm ${ }^{(10,11)}$ was observed using 150 and 350 particles, and results are shown in Figs. 6 and 7.

From the simulation results in Figs. 6 and 7, the real trajectory of the moving target was used to induce the turning model motion in the three-dimensional plane, which was simulated by 150 and 350 particles, respectively. The filtered trajectory of the PF algorithm was consistent with the real trajectory. The error of 350 particles varied between 0 and $9 \mathrm{~m}$, while the error of 150 particles varied between 0 and $13 \mathrm{~m}$. The tracking accuracy of the PF algorithm with 350 particles was higher than that with of 150 particles. Therefore, when the PF algorithm was used to track moving targets, the higher the number of particles, the higher the positioning tracking accuracy.

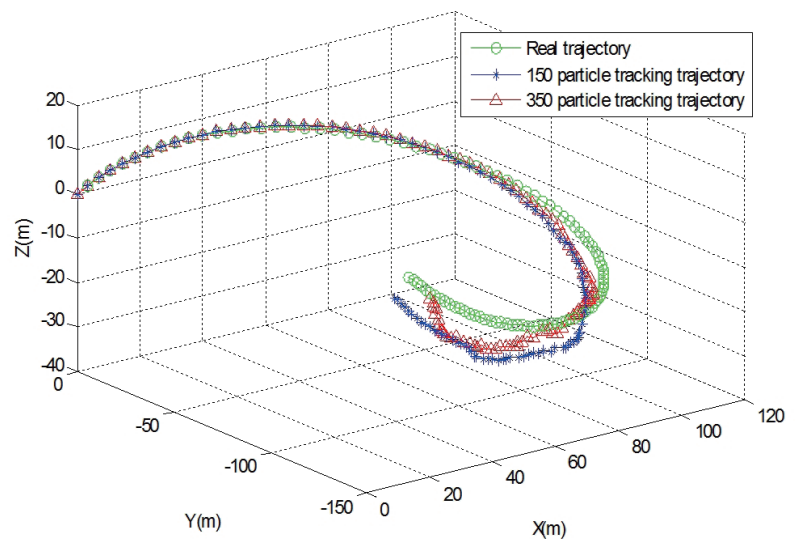

Fig. 6. (Color online) Location motion track.

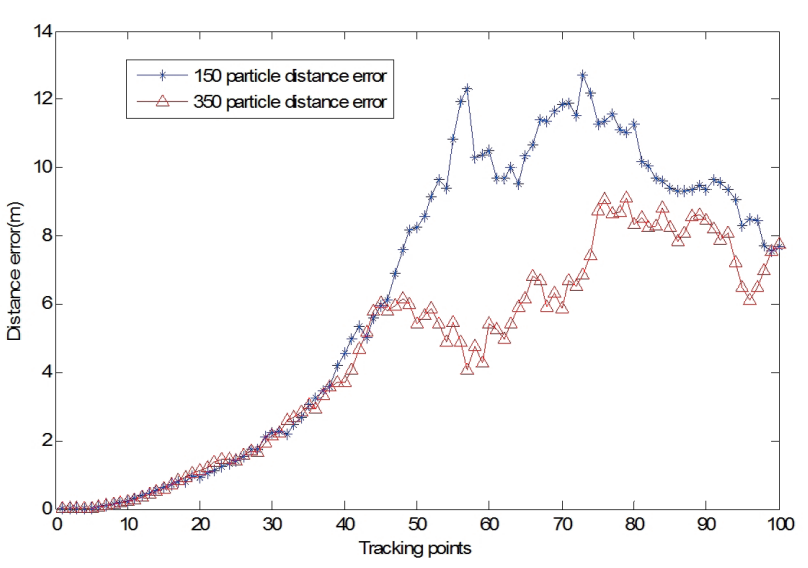

Fig. 7. (Color online) Location error. 


\section{Conclusions}

In this study, the design process of a dual-frequency microstrip antenna was analyzed in detail, and the microstrip antenna capable of receiving GPS and DTMB signals simultaneously was simulated. Simulation results indicate that the antenna has good radiation characteristics and high practicability. These simulation results provided a stable foundation for further study of the feasibility of the combined navigation positioning model based on GPS and DTMB. In addition, the dynamic tracking effect of the PF algorithm for an integrated navigation positioning system was also studied, and simulation results showed that the filter tracking trajectory is close to the real trajectory, and the higher the number of particles, the higher the positioning accuracy. These results proved the feasibility of using GPS and DTMB for combined positioning.

\section{Acknowledgments}

This work was supported financially by Yunnan Province Science and Technology Department and Education Department Project (2017FH001-067, 2017FH001-117, and 2016ZDX127), China. Also, we would like to thank Professors Fei Zhou and Jianxing Shao for their guidance.

\section{References}

1 G. B. Moon, G. I. Jee, and J. G. Lee: Int. J. Control Autom. Syst. 9 (2011) 574. https://doi.org/10.1007/s12555011-0318-9

2 L. L Dai, Z. C. Wang, and Z. X. Yang: IEEE Commun. Mag. 50 (2012) 150. https://doi.org/10.1109/ MCOM.2012.6211500

3 C. M. Cheng, S. H. Tseng, and W. S. Chen: Sens. Mater. 29 (2014 ) 491. https://doi.org/10.18494/SAM.2017.1531

4 S. Roy and U. Chakraborty: Int. Commun. 12 (2018) 1448. https://doi.org/10.1049/iet-com.2018.0170

5 V. Sathi, N. Ehteshami, and J. Nourinia: IEEE Antennas Wirel. Propag. Lett. 11 (2012) 1018. https://doi. org/10.1109/LAWP.2012.2215001

6 H. A. Majid, M. K. A. Rahim, M. R. Hamid, and M. F. Ismail: IEEE Antennas Wirel. Propag. Lett. 11 (2012) 616. https://doi.org/10.1109/LAWP.2012.2202869

7 A. F. Sheta, A. Mohr, and S. F. Mahmoud: Microwave Opt. Tech. Lett. 35 (2002) 363. https://doi.org/10.1002/ mop. 10608

8 M. Nasiri, H. Mirzajani, E. Atashzaban, and H. B. Ghavifekr: Wirel. Pers. Commun. 72 (2013) 259. https://doi. org/10.1007/s11277-013-1012-4

9 D. Caratelli, R. Cicchetti, G. Bit-Babik, and A. Faraone: IEEE Trans. Antennas Propag. 54 (2006) 1871. https:// doi.org/10.1109/TAP.2006.874364

10 P. Banerjee, O. Karpenko, L. Udpa, M. Haq, and Y. M. Deng: Compos. Struct. 194 (2018) 527. https://doi. org/10.1016/j.compstruct.2018.04.033

11 M. G. Gan, Y. L. Cheng, Y. A. Wang, and J. Chen: J. Sys. Eng. Electron. 27 (2016) 51. https://doi.org/10.1109/ JSEE.2016.00006 


\section{About the Authors}

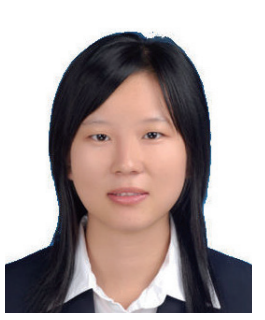

An-Hong Tian received her B.S. and M.S. degrees from Chongqing University of Posts and Telecommunications, China, in 2007 and 2010, respectively. From 2011 to 2016, she was a lecturer at Qujing Normal University, China. Since 2017, she has been an associate professor at Qujing Normal University. Her research interests are in remote sensing, wireless navigation, and artificial intelligence. (tianah@mail.qjnu.edu.cn)

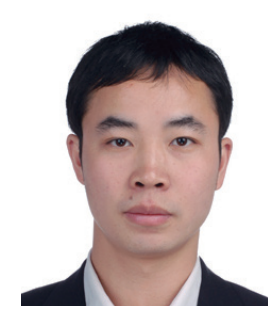

Cheng-Biao Fu received his B.S. and M.S. degrees from Chongqing University of Posts and Telecommunications, China, in 2005 and 2009, respectively. Since 2015, he has been a lecturer at Qujing Normal University. His research interests are in information identification and processing, hyperspectral remote sensing, and Internet of Things.

(fucb@mail.qjnu.edu.cn)

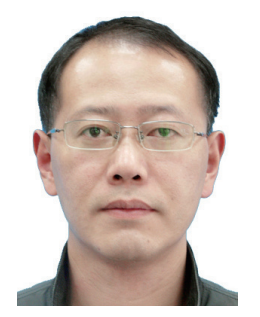

Shun-Ji Zhang received his B.S. degree from Yunnan Normal University, China, in 1995. He received his M.S. degree from Yunnan University, China, in 2009. Since 2006, he has been an associate professor at Qujing Normal University. His research interest is in Internet of Things. (tianfucb@163.com) 